\title{
Proteasome Modulator 9 SNPs are linked to hypertension in type 2 diabetes families
}

Claudia Gragnoli, 1,2,3

\begin{abstract}
Background: Chromosome 12q24 was recently associated with hypertension. Proteasome Modulator 9 (PSMD9) lies in the 12q24 locus and is in linkage with MODY3, type 2 diabetes (T2D), microvascular and macrovascular pathology, carpal tunnel syndrome, and hypercholesterolemia in Italian families.

Aims: Our goal was to determine whether PSMD9 is linked to elevated blood pressure/hypertension in T2D families.

Methods: We characterized the Italian T2D families' members for presence and/or absence of elevated blood pressure $(\geq 130 / 80)$ and/or hypertension. The phenotypes were described as unknown in all cases in which the diagnosis was either unclear or the data were not available for the subject studied. We tested in the 200 Italians families for the presence of the linkage of the PSMD9 T2D risk single nucleotide polymorphisms (SNPS) IVS3+nt460 $A>G$, IVS3+nt437 T > C and E197G A > G with elevated blood pressure/hypertension. The non-parametric linkage analysis was performed for this qualitative phenotype by using the Merlin software; the Lod score and correspondent P-value were calculated. Parametric linkage analysis was also performed. For the significant linkage score, 1000 replicates were run to calculate the empirical P-value.
\end{abstract}

Results: The PSMD9 gene SNPS studied are in linkage with elevated blood pressure/hypertension in our Italian families.

Conclusions: We conclude that the PSMD9 gene and/or any variant in linkage disequilibrium with the SNPS studied contribute to the linkage to hypertension within our family dataset. This is the first report of PSMD9 linkage to hypertension within the 12 q24 locus.

\section{Introduction}

A recent study has shown that changes in retinal vascular caliber are linked to the chromosome 12q24 locus in a large Caucasian population [1]. Microcirculation is important in determination of hypertension [1] and retinal vascular changes reflect early microvascular disease and predict cardiovascular events. In two independent samples, the locus $12 \mathrm{q} 24$ was also associated with coronary heart disease and hypertension [1]. Thus, the chromosome 12q24 locus carries at least a gene contributing to hypertension. In this locus lies Proteasome Modulator 9 (PSMD9), a coactivator of insulin gene transcription, which is highly

\footnotetext{
Correspondence: claudia.gragnoli@gmail.com

'Laboratory of Molecular Genetics of Complex and Monogenic Disorders,

Department of Medicine and Cellular \& Molecular Physiology and

Biostatistics, Penn State University and M. S. Hershey Medical Center,

Hershey, PA, USA

Full list of author information is available at the end of the article
}

expressed in pancreatic islets [2]. PSMD9 is a ubiquitous protein; therefore its biological role may be broad. PSMD 9 overexpression cause beta-cell dysfunction and contribute to type 2 diabetes (T2D) [2]. We reported that PSMD9 may rarely cause T2D by unique mutations [3]. We identified a significant linkage of the PSMD $A / T / G$ haplotype to late-onset $\mathrm{T} 2 \mathrm{D}$, with the strongest evidence under the recessive model [4], and to MODY3 under the additive model [5] in Italians. The contribution of intronic variants to complex disorders is an accepted concept. We recently reported a linkage of the PSMD9 T2D risk single nucleotide polymorphisms (SNPs) with T2D-nephropathy [6], T2D-neuropathy [7], retinopathy [8], carpal tunnel syndrome [9], hypercholesterolemia [10], and macrovascular pathology [11]. Given the reported linkage data of PSMD9 within the locus 12q24, and the evidence of linkage with microcirculation within the same locus [1], PSMD9 is a candidate gene for hypertension. Further, as PSMD9 is

\section{Biomed Central}

(c) 2011 Gragnoli; licensee BioMed Central Ltd. This is an Open Access article distributed under the terms of the Creative Commons Attribution License (http://creativecommons.org/licenses/by/2.0), which permits unrestricted use, distribution, and reproduction in any medium, provided the original work is properly cited. 
linked to T2D, it should be screened to identify any inheritance contributing factors to the T2D-associated phenotypes, of which hypertension is a major player.

In the present study, we aimed at testing the PSMD9 $I V S 3+n t 460, I V S 3+n t 437, E 197 G$ T2D risk SNPs for linkage with elevated blood pressure and/or hypertension in our 200 Italian T2D families.

\section{Methods}

\section{Ethical Statement}

the subjects were all recruited from center Italy following the Helsinki declaration guidelines. Subjects gave written informed consent. The Penn State College of Medicine Ethical Committee approved the study.

\section{Families}

We recruited 200 Italian T2D affected siblings and extended families, including also unaffected members. The families originate from the center of Italy. The members are at least three generations Italians. Identical twins were excluded. These T2D families have been helpful in the whole or as unrelated T2D cases in identifying or excluding diabetes risk genes and/or variants in previous studies [12-24]. We characterized the Italian families for presence/absence of elevated blood pressure/hypertension [presence is considered by blood pressure $\geq 130 / 80 \mathrm{mmHg}$ in drug-naïve patients or by use of anti-hypertensive medication(s)]. Phenotype is described as unknown if diagnosis is unclear or data are lacking. Most subjects with T2D have elevated blood pressure and few subjects without T2D have elevated blood pressure. The total of the subjects available for the analysis including the ungenotyped parents is 928 (443 founders, 485 non-founders; 467 females, 461 males) with an average family members of 4.62 . The diagnostics of hypertension is present in 373 genotyped individuals, with a prevalence of $94.6 \%$.

\section{Sequencing}

Via PCR, we amplified the IVS3 PSMD9 region containing the $+n t 460 A>G$ and $+n t 437 C>T$ SNPs and the exon 5 coding region containing the E197G $A>G$ SNP with specific primers in the affected and unaffected family members. We directly sequenced the PCR products, status post-purification via EXOSAP-IT, on an automated ABI 3730 Sequencer.

\section{Statistical Analysis}

We tested in the 200 Italian families for linkage of the PSMD9 SNPs with elevated blood pressure/hypertension. Both non-parametric and parametric linkage analysis for the qualitative phenotype were performed for the three SNPs via Merlin software [25]. Allele frequencies were calculated from the data [25]. Merlin analysed all genetically informative families $(\mathrm{n}=129$; total subjects $=$ 596) within this dataset, depending on the presence of both genotypes and hypertension phenotype in families whose structure, given the data, was amenable to the linkage tests. The families analysed by Merlin are representative of the population. We previously reported that the PSMD9 SNPs IVS3+nt460, IVS3+nt437, E197G are in strong linkage disequilibrium (LD) [3]. The following parameters were used for the parametric linkage analysis based on the SNPs-cluster, thus eliminating the LD inflation of the linkage signal: disease allele frequency 0.25 , dominant model with penetrance for homozygous non-risk allele 0.31 (equal to the prevalence of hypertension in the Italian population), for heterozygous risk allele 1.00, for homozygous risk allele 1.00; recessive model with penetrance for homozygous non-risk allele 0.31 , for heterozygous risk allele 0.31 , for homozygous risk allele 1.00; additive model with penetrance for homozygous non-risk allele 0.31 , for heterozygous risk allele 0.45 and for homozygous risk allele 0.90. All results are reported as LOD scores calculated by Merlin.

For each test performed, to exclude the presence of any false positive in our results, we calculated how many times similar P-values were expected by chance in 1,000 replicates of simulations by using the gene dropping method: this analysis replaces real data with simulated data, while maintaining the pedigree structure, allele frequencies and recombination fraction. These datasets are generated under the null hypothesis of no linkage.

\section{Results}

The results of the non-parametric and parametric linkage analysis performed for the qualitative phenotype hypertension are reported in table 1 . Both analytical methods have reported significant results with the most significant being for the non-parametric model. The PSMD9 SNP IVS3+nt460 is slightly more significant than the other two SNPs. Under the parametric model analysis, the most significant is the dominant model. The simulation analyses of 1,000 replicates have excluded false positives and establish the validity of the real data.

\section{Discussion}

Our analysis show that the PSMD9 IVS3 +nt460 A > G and $+n t 437 C>T$ and exon 5 E197G $A>G$ SNPs studied and/or any variants in LD with them are in linkage with elevated blood pressure/hypertension in our 200 Italian families. The PSMD9 SNPs studied contribute to the linkage of the reported phenotype. It is possible that the recessive and additive models appear less significant than the dominant model, as the disease penetrance value given for the heterozygous state under both models, and for the homozygous state under the additive model, is inferior to 1 . Thus, some of the power of the data may be 
Table 1 Non-parametric and Parametric Linkage Analysis of Hypertension in the 200 Italian Families by Merlin software

\begin{tabular}{lccccc}
\hline Phenotype & Prevalence & Families & Lod Score & P & Empirical P \\
\hline Non-Parametric & $94.60 \%$ & 129 & 3.32 & 0.00005 & 0.001 \\
\hline Dominant Model; SNPs Cluster & $94.60 \%$ & 129 & 2.282 & 0.001202 & 0.000 \\
\hline Recessive Model; SNPs Cluster & $94.60 \%$ & 129 & 1.979 & 0.002561 & 0.000 \\
\hline Additive Model; SNPs Cluster & $94.60 \%$ & 129 & 0.926 & 0.039048 & 0.000 \\
\hline
\end{tabular}

Prevalence $=$ phenotype prevalence among the family subjects studied; Families = families number analyzed; Lod score $=$ derived from the non-parametric linkage analysis or the parametric analysis cluster-based by Merlin; $P=p$-value; Empirical $P=p$-value derived from 1000 replicates by using the gene dropping method. Hypertension is indicated as presence of blood pressure $\geq 130 / 80$ in absence of medications or presence at least of one anti-hypertensive drug.

lost. On the other hand, recessive and additive models are still significant as the variants in homozygous state are likely inherited by the affected family subjects more often than expected by the null hypothesis of no linkage and thus contribute to the linkage signal. The limitation of our study is that the linkage may capture the signal from the potential co-inheritance of genetic variants linked to another phenotype and to the underlying T2D that is commonly shared with hypertension; however, as T2D patients have high prevalence of hypertension, virtually all linkage studies performed in T2D may as well mask a linkage with hypertension. In fact, when we statistically analyze only a single phenotype, we cannot protect the results from any potentially known and unknown associated factor with the phenotype under study and from the co-inheritance of genetic variants related to that factor underlying the linkage signal.

The strength of our study resides in the significant data both at the level of non-parametric analysis, which is not vulnerable to miscalculation of allele frequencies and penetrance values, as well as at the level of the parametric analysis, which may suffer from not perfect estimates of allele frequencies and penetrance values.

However, the best statistical power test is the simulation empirical P-value, and in this study all empirical P-values have excluded the possibility of false positives in our data analysis.

These findings have not yet been confirmed in other ethnicities.

\section{Conclusions}

The PSMD9 SNPs IVS3+nt460, IVS3+nt437, E197G are significantly linked to elevated blood pressure/hypertension in the Italian T2D family dataset.

\section{Abbreviations}

PSMD9: Proteasome Modulator 9; SNP: single nucleotide polymorphism; T2D: type 2 diabetes; MODY3: maturity-onset diabetes of the young 3; IVS: intervening sequence (intron)

\section{Acknowledgements}

Special thanks go to the Molecular Biology staff of Bios Biotech MultiDiagnostic Health Center (Rome, Italy), which provided technical support for this study. This study was supported by Penn State College of Medicine.

\section{Author details}

${ }^{1}$ Laboratory of Molecular Genetics of Complex and Monogenic Disorders, Department of Medicine and Cellular \& Molecular Physiology and Biostatistics, Penn State University and M. S. Hershey Medical Center, Hershey, PA, USA. ${ }^{2}$ Center for Biotechnology and Department of Biology, Temple University's College of Science \& Technology, Philadelphia, PA, USA ${ }^{3}$ Molecular Biology Laboratory, Bios Biotech Multi-Diagnostic Health Center, Rome, Italy.

\section{Authors' contributions}

CG conceived and designed the study, collected the clinical information, performed the statistical analysis and drafted the manuscript. CG also read and approved the final manuscript.

\section{Competing interests}

The authors declare that they have no competing interests.

Received: 10 August 2011 Accepted: 28 August 2011

Published: 28 August 2011

\section{References}

1. Ikram MK, Xueling S, Jensen RA, Cotch MF, Hewitt AW, Ikram MA, Wang J,, Klein R, Klein BE, Breteler MM, Cheung N, Liew G, Mitchell P,

Uitterlinden AG, Rivadeneira F, Hofman A, de Jong PT, van Duijn CM, Kao L, Cheng CY, Smith AV, Glazer NL, Lumley T, McKnight B, Psaty BM, Jonasson F, Eiriksdottir G, Aspelund T, Harris TB, Launer LJ, Taylor KD, Li X, lyengar SK, Xi Q, Sivakumaran TA, Mackey DA, Macgregor S, Martin NG, Young TL, Bis JC, Wiggins KL, Heckbert SR, Hammond CJ, Andrew T, Fahy S, Attia J, Holliday EG, Scott RJ, Islam FM, Rotter Jl, McAuley AK, Boerwinkle E, Tai ES, Gudnason V, Siscovick DS, Vingerling JR, Wong TY: Four novel Loci $(19 q 13,6 q 24,12 q 24$, and 5q14) influence the microcirculation in vivo. PLoS Genet 2010, 6:e1001184.

2. Thomas MKYK, Tenser MS, Wong GG, Habener JF: Bridge-1, a novel PDZdomain coactivator of E2A-mediated regulation of insulin gene transcription. Mol Cell Biol 1999, 19:8492.

3. Gragnoli C, Cronsell J: PSMD9 gene variants within NIDDM2 may rarely contribute to type 2 diabetes. J Cell Physiol 2007, 212:568.

4. Gragnoli C: PSMD9 gene in the NIDDM2 locus is linked to type 2 diabetes in Italians. J Cell Physiol 2010, 222:265.

5. Gragnoli C: PSMD9 is linked to MODY3. J Cell Physiol 2010, 223:1.

6. Gragnoli C: T2D-nephropathy linkage within 12q24 locus. Diabetes Res Clin Pract 2011, 92:e73.

7. Gragnoli C: PSMD9 is linked to type 2 diabetes neuropathy. J Diabetes Complications 2011.

8. Gragnoli C: Proteasome Modulator 9 gene is linked to diabetic and nondiabetic retinopathy in T2D. Ophthalmic Genet 2011.

9. Gragnoli C: Proteasome Modulator 9 and Carpal Tunnel Syndrome. Diabetes Res Clin Pract 2011 August 19.

10. Gragnoli C: Hypercholesterolemia and a candidate gene within the 12q24 locus. Cardiovasc Diabetol 2011, 10:38.

11. Gragnoli C: Proteasome modulator 9 and macrovascular pathology of T2D. Cardiovasc Diabetol 2011, 10:32.

12. Gragnoli C: Kir6.2 (KCNJ11) E23K variant in Type 2 Diabetes in Italians. Obesity and Metabolism 2007, 3:97.

13. Gragnoli C: CHOP T/C and C/T haplotypes contribute to early-onset type 2 diabetes in Italians. J Cell Physiol 2008, 217:291. 
14. Gragnoli C, Cronsell J: PPARY P12A variant in Type 2 Diabetes in Italians. Obesity and Metabolism 2007, 3:99.

15. Gragnoli C, Cockburn BN, Chiaramonte F, Gorini A, Marietti G, Marozzi G, Signorini AM: Early-onset Type II diabetes mellitus in Italian families due to mutations in the genes encoding hepatic nuclear factor 1 alpha and glucokinase. Diabetologia 2001, 44:1326.

16. Gragnoli C, Cronsell J: Meta-analysis of the IPF1 D76N polymorphism in a worldwide type 2 diabetes population. Minerva Med 2007, 98:163.

17. Gragnoli C, Lindner T, Cockburn BN, Kaisaki PJ, Gragnoli F, Marozzi G, Bell Gl: Maturity-onset diabetes of the young due to a mutation in the hepatocyte nuclear factor- 4 alpha binding site in the promoter of the hepatocyte nuclear factor-1 alpha gene. Diabetes 1997, 46:1648.

18. Gragnoli C, Menzinger Von Preussenthal G, Habener JF: Triple genetic variation in the HNF-4alpha gene is associated with early-onset type 2 diabetes mellitus in a philippino family. Metabolism 2004, 53:959.

19. Gragnoli C, Milord E, Cox N: Worldwide metanalysis of NEUROG3 variants in type 2 diabetes. Obesity and Metabolism 2006, 2:68.

20. Gragnoli C, Milord E, Habener JF: Linkage study of the glucagon receptor gene with type 2 diabetes mellitus in Italians. Metabolism 2005, 54:786.

21. Gragnoli C, Pierpaoli L, Piumelli N, Chiaramonte F: Linkage studies for T2D in Chop and C/EBPbeta chromosomal regions in Italians. J Cell Physiol 2007, 213:552.

22. Gragnoli C, Stanojevic V, Gorini A, Von Preussenthal GM, Thomas MK, Habener JF: IPF-1/MODY4 gene missense mutation in an Italian family with type 2 and gestational diabetes. Metabolism 2005, 54:983.

23. Milord $\mathrm{E}$, Gragnoli $\mathrm{C}$ : Chromosome 10 locus in linkage to type 2 diabetes in Italians. Obesity and Metabolism 2007, 3:44.

24. Milord E, Gragnoli C: NEUROG3 variants and type 2 diabetes in Italians. Minerva Med 2006, 97:373.

25. Abecasis GR, Cherny SS, Cookson WO, Cardon LR: Merlin-rapid analysis of dense genetic maps using sparse gene flow trees. Nat Genet 2002, 30:97.

doi:10.1186/1475-2840-10-77

Cite this article as: Gragnoli: Proteasome Modulator 9 SNPs are linked to hypertension in type 2 diabetes families. Cardiovascular Diabetology 2011 10:77

\section{Submit your next manuscript to BioMed Central and take full advantage of:}

- Convenient online submission

- Thorough peer review

- No space constraints or color figure charges

- Immediate publication on acceptance

- Inclusion in PubMed, CAS, Scopus and Google Scholar

- Research which is freely available for redistribution

Submit your manuscript at www.biomedcentral.com/submit 